\title{
AISLAMIENTO E IDENTIFICACIÓN DE Listeria spp. A PARTIR DE MUESTRAS DE PESCADO FRESCO EXPENDIDO EN PAMPLONA (NORTE DE SANTANDER)
}

\section{ISOLATION AND IDENTIFICATION OF Listeria spp. FROM FRESH FISH SAMPLES, MARKETED IN PAMPLONA (NORTE DE SANTANDER)}

\author{
Fanny Herrera A. ${ }^{1}$, William Suárez Q. ${ }^{2}$
}

\begin{abstract}
${ }^{1}$ Ph.D. Universidad de Pamplona. Docente Titular. Departamento de Microbiología, Facultad de Ciencias Básicas. Grupo de Investigación en Microbiología y Biotecnología (GIMBIO). Ciudad Universitaria. Pamplona, Norte de Santander. Colombia. Correspondencia: fannyh@unipamplona.edu.co; ${ }^{2}$ Microbiólogo con Énfasis en alimentos, M.Sc. en Ciencia y Tecnología de Alimentos, Universidad de Pamplona. Docente catedrático. Departamento de Microbiología, Facultad de Ciencias Básicas. e-mail:aquifex3@hotmail.com
\end{abstract}

Rev. U.D.C.A Act. \& Div. Cient. 15(2): 257 - 265, 2012

\section{RESUMEN}

El género Listeria contiene ocho especies, destancándose Listeria monocytogenes patógena para el hombre que puede ocasionar gastroenteritis, meningitis, neuro-encefalitis, septicemia, abortos e, incluso, la muerte. L. monocytogenes es una bacteria ubicua que se puede aislar a partir de los ecosistemas donde habitan los peces, logrando llegar al producto terminado e inclusive, a concentraciones elevadas, cuando el pescado se procesa empleando inadecuadas prácticas de limpieza y de desinfección o se conserva durante mucho tiempo a temperaturas de refrigeración, debido a que es un microorganismo psicrotrófico. Los objetivos del presente estudio fueron determinar la presencia de Listeria spp. en muestras de pescado fresco expendido en la plaza de mercado de la ciudad de Pamplona, comparando el método tradicional y un inmunocromatográfico y determinar si existe correlación entre la presencia de Escherichia coli y Listeria spp., en las muestras. Se aislaron $L$. monocytogenes, $L$. innocua y $L$. grayi, con prevalencias del $3,9 \%, 17,6 \%$, y $13,7 \%$, respectivamente, con una incidencia total del $35,3 \%$. Al comparar los dos métodos de aislamiento, se encontró que no se existieron diferencias significativas entre ellos. Finalmente, se determinó que la presencia de E. coli es un buen indicador de la manifestación de cepas de Listeria en las muestras. La presencia de $L$. monocytogenes en las muestras indica que el pescado comercializado en la plaza de mercado de Pamplona puede ser un vehículo importante para este patógeno e, implica, que este alimento puede representar un riesgo para la salud de los consumidores, especialmente, para población vulnerable.
Palabras clave: Listeria monocytogenes, pescado, Escherichia coli, prevalencia.

\section{SUMMARY}

The genus Listeria comprises eight species between them Listeria monocytogenes, pathogenic to humans, causing gastroenteritis, meningitis, neuro-encephalitis, septicemia, abortions and even death. L. monocytogenes is an ubiquitous bacterium which can be isolated from ecosystems inhabited by fish, reaching the finished product, even at high concentrations, when the fish is processed using inadequate cleaning and disinfection practices and/or is stored for a long time at refrigeration temperatures because it is psychrotrophic. The objectives of this study were to determine the presence of Listeria spp. in fresh fish samples expended in the market place of Pamplona, comparing the traditional method with an immunochromatografic one, and to verify whether there is a correlation between the presence of Escherichia coli and Listeria spp. in the samples. L. monocytogenes, L. innocua and $L$. grayi were isolated, with prevalences of $3.9 \%$, $17.6 \%$ and $13.7 \%$, respectively, with an overall incidence of $35.3 \%$. By comparing the isolation methods, traditional and immunochromatografic, no significant differences between them were found. Finally, it was established that the presence of E.coli is a good indicator of the presence of strains of Listeria in the samples. The presence of $L$. monocytogenes in the samples indicates that fish sold in the market place of Pamplona can be an important vehicle for this pathogen and implies, that this food might pose a health risk to consumers, especially the vulnerable population. 
Key words: Listeria monocytogenes, fish, Escherichia coli, prevalence.

\section{INTRODUCCIÓN}

En la actualidad, se reconocen ocho especies de Listeria: L. monocytogenes: L. seeligeri, L. ivanovii, L. innocua, L. welshimeri, $L$. grayi y dos nuevas especies $L$. marthii y $L$. rocourtiae (Orsi et al. 2011). De las ocho, L. monocytogenes es la que tiene importancia, desde el punto de vista de la salud pública (Chen et al. 2010a).

L. monocytogenes puede ocasionar dos tipos de síndromes en el hombre. En primer lugar, listeriosis, caracterizado por síntomas y por signos severos y se presenta en población vulnerable y, en menor medida, síndrome de tipo gastrointestinal, leve autolimitante, que puede afectar a la población en general (Todd \& Notermans, 2011). La mayoría de las infecciones por $L$. monocytogenes se dan en personas con un sistema inmune deficitario por la edad, por enfermedades como el cáncer, el sida, la cirrosis, por transplante de órganos, entre otros. La bacteria puede atravesar la barrera intestinal y diseminarse desde los nódulos linfáticos mesentéricos hacia el hígado y el bazo, desde los cuales, puede alcanzar el cerebro o la placenta (Orsi et al. 2011). Los síntomas en los casos perinatales pueden variar desde fiebre en la madre, acompañada de síntomas de gastroenteritis; en general, el feto no se afecta gravemente, pero se puede presentar septicemia y, en los casos más graves, meningitis (Yucel \& Balci, 2010). Puede ocasionar abortos, partos prematuros o el nacimiento de un bebé muerto. En los casos que no implican gestación, la bacteria tiene tropismo hacia el sistema nervioso central, presentando meningitis y meningoencefalitis. El porcentaje de mortalidad por esta bacteria es muy alta en los grupos de riesgo, del $20-40 \%$ (Huss et al. 2003). El periodo de incubación de la enfermedad en personas adultas varía entre una y varias semanas; la dosis infecciosa se desconoce, aunque se asume que menos de 100 células viables $/ \mathrm{g} / \mathrm{mL}$, pueden ser infectivas (Rocourt et al. 2000).

Listeria es una bacteria anaerobia facultativa; crece a una temperatura óptima $37^{\circ} \mathrm{C} ; L$. monocytogenes es psicrotrófica logrando sobrevivir a varios grados bajo cero, a $\mathrm{pH}$ entre 4,6 a 9,5 y a una actividad de agua $\left(\mathrm{a}_{\mathrm{w}}\right)$ baja, como 0,92 (Carpentier \& Cerf, 2011), incluso, puede crecer en concentraciones de sal, hasta del 30\% (Wan Norhana et al. 2010).

El pescado aporta nutrientes muy importantes en la dieta del ser humano y además provee reconocidos beneficios para la salud (Yucel \& Balci, 2010). El pescado fresco posee ciertas características que favorecen el crecimiento de Listeria, como son $\mathrm{pH}$, entre 6,6-6,8; $\mathrm{a}_{\mathrm{w}}$, entre 0,98-0,99; potencial de óxido-reducción, entre +100 a $+>300$ mv (Ross et al. 2000) y posibles largos periodos de tiempo, conservado a temperaturas de refrigeración (Motarjemi \& Adams, 2006). Se estima que un 7,0\% del pescado crudo contiene Listeria monocytogenes; adicionalmente, las especies de Listeria al ser ubicuas, se logran aislar de ríos, de mar y de aguas residuales, así que la oportunidad de contaminación cruzada con el ambiente de proceso del pescado es alta (Otto et al. 2011).

Se ha determinado una incidencia anual de listeriosis alimentaria de 2.493 casos y 430 muertes, en Estados Unidos (Todd \& Notermans, 2011); a pesar de esta incidencia, se encuentra entre las más frecuentes causas de muerte ocasionadas por Enfermedades de Origen Alimentario (ETAs) (Lomonaco et al. 2011). En el caso del pescado, se han reportado varios brotes por el consumo de pescado o derivados; en 1980, se reportó el primer brote, que afectó a 29 individuos y ocasionó nueve muertes; posteriormente, se reportó otro caso, debido al consumo de pescado en Italia (Elliot \& Kvenberg, 2000); en Nueva Zelanda, se informaron de 22 casos de listeriosis, que afectaron a mujeres embarazadas, ocasionando siete muertes (Motarjemi \& Adams, 2006); en Francia, se rechazó un lote de pescado procedente de China, por la presencia de L. mononocytogenes (Rocourt et al. 2000).

En Lationamérica, en 1998, se presentó el primer registro acerca la presencia de Listeria en pescado, concretamente en mariscos; posteriormente, se han reportado aislamientos de L. monocytogenes en Brasil, en Costa Rica, en Trinidad, en Chile y en Argentina (Destro, 2000).

A pesar de la baja incidencia de listeriosis, la evidencia epidemiológica demuestra claramente que el pescado y sus productos son responsables de ETAs por $L$. monocytogenes (Rocourt et al. 2000).

En Colombia, existen pocos datos sobre la epidemiología de la listeriosis, debido, principalmente, a que no es una enfermedad de notificación obligatoria; en un estudio publicado, realizado en 1994, en un hospital de tercer nivel (Cali, Valle), se informó sobre 19 casos clínicos de listeriosis, diez, en adultos inmunosuprimidos; dos, en mujeres embarazadas; seis, en neonatos y, un caso, en una adolescente de doce años (Crespo et al. 1999). Se considera que el número de listeriosis en Colombia va en aumento, debido al incremento dentro de la población de enfermedades que deprimen el sistema inmune, como cáncer, sida, hepatitis o transplantes de órganos (Medrano et al. 2006).

En nuestro país, el pescado ocupó el quinto lugar dentro de los principales alimentos implicados en ETAs, en el periodo 
1998-2003, siendo L. monocytogenes el cuarto género bacteriano, involucrado en las mismas (Muriel, 2008).

El protocolo tradicional para la identificación de Listeria es muy laborioso y prolongado en el tiempo, ya que implica dos enriquecimientos: una posterior siembra en medios selectivos- diferenciales y la identificación bioquímica (Norma Técnica Colombiana, NTC 4666), razones por las cuales, se están implementado métodos que eviten jornadas intensivas de trabajo para el personal con resultados, en menor tiempo. Dentro de ellos, se cuenta con el método inmunocromatográfico, que usa un dispositivo de flujo lateral; el dispositivo de la prueba cuenta con una membrana porosa de nitrocelulosa, en la que es inmovilizado un anticuerpo marcado con oro, el cual, se une específicamente y captura un antígeno particular de Listeria, si está presente en la muestra; este método se considera altamente sensible y específico (McMeekin, 2003).

Los coliformes totales son bacilos Gram negativos asporógenos, que fermentan la lactosa en 24-48 horas, a $37^{\circ} \mathrm{C}$, dentro de los cuales, se encuentra Escherichia coli, que además tiene la particularidad de fermentar la lactosa con producción de gas, entre $44-46^{\circ} \mathrm{C}$ y se le considera el microorganismo coliforme fecal por excelencia, aunque algunas cepas de Citrobacter y de Klebisella, se ajustan a este comportamiento (Jay et al. 2009). Escherichia coli es el indicador de contaminación fecal en los alimentos más utilizado, ya que constituye el $10 \%$ de los microorganismos intestinales de los seres humanos y otros animales de sangre caliente (Marín et al. 2009).

Los objetivos de este estudio fueron, en primer lugar, aislar e identificar cepas de Listeria spp., a partir muestras de pescado fresco, expendido en Pamplona (Norte de Santander), comparando el método tradicional con el inmunocromatográfico y, en segundo lugar, determinar si existe correlación entre la presencia de E. coli y Listeria spp., en las muestras.

\section{MATERIALES Y MÉTODOS}

Número de muestras: Se realizó una encuesta, aplicando un muestreo para proporciones, con el fin de determinar qué tan frecuente es el consumo de pescado en la ciudad de Pamplona. Los resultados revelaron que del total de la población entrevistada, correspondiente a 256 personas, 163 personas consumen pescado y, de ellas, 73 , lo adquieren en la plaza de mercado de la ciudad; 35, en supermercados; 40 , ventas callejeras y 15 , ventas detalladas. Teniendo en cuenta que la gran mayoría de las personas compran el pescado en la plaza de mercado, se decidió muestrear el pescado expendido en este lugar; con el dato de 73 personas, se aplicó un muestreo para proporciones, con un nivel de confianza del $95 \%$, error del 7,4\% y aplicando la fórmula del muestreo para proporciones con población finita, el resultado fue de 51 muestras.

El tipo de estudio aplicado en este trabajo fue descriptivo. El método de muestreo fue intencional no probabilístico.

Muestras: Se analizaron un total de 51 muestras distribuidas así: Bagre, 20 (Brachyplatistoma spp.); trucha, 12 (Oncorhynchus mykiss); bocachico, 12 (Prochilodus magdalenae) y mojarra, 7 (Oreochromis spp.). Estas especies de pescado fueron seleccionadas, debido a la preferencia que reflejaron los consumidores en la encuesta. Se tomaron $100 \mathrm{~g}$ de cada muestra que fueron trasladadas al laboratorio en bolsas estériles, en nevera portátil y fueron analizadas inmediatamente después de su recolección.

Aislamiento de Listeria spp.: Para el aislamiento tradicional, se siguió la metodología establecida por la Norma Técnica Colombiana 4666 (1999): Método Horizontal para la Detección de L. monocytogenes. El método alterno de detección empleado fue el inmunocromatográfico, el cual, es un método de detección inmunológico basado en el principio "immune flow", que utiliza anticuerpos marcados con oro. Se utilizó el Singlepath ${ }^{\mathrm{TM}}$ Listeria, siguiendo las instrucciones del fabricante así: se pesaron $25 \mathrm{~g}$ de la muestra, en $225 \mathrm{~mL}$ del caldo Fraser media concentración, incubando a $30^{\circ} \mathrm{C} / 18-24 \mathrm{~h}$. Luego, se transfirieron $0,1 \mathrm{~mL}$ en $9,9 \mathrm{~mL}$ de Caldo UVM, incubando a $30^{\circ} \mathrm{C} / 18-24$ h. Posteriormente, se transfirieron de $1-2 \mathrm{~mL}$ del cultivo enriquecido a un tubo de polipropileno. Incubando a $80^{\circ} \mathrm{C} / 20$ min y dejando enfriar a temperatura ambiente $\left(18-26^{\circ} \mathrm{C}\right)$. Se tomaron $160 \mu \mathrm{L}$ del cultivo calentado y enfriado, dispensándolo en el orificio circular del dispositivo. Se observaron los resultados a los 20 min de aplicar la muestra en el dispositivo (Merck, 2008).

Se confirmaron las cepas positivas, sembrando por aislamiento sobre el agar Palcam; posteriormente, se transfirieron las colonias típicas sobre Agar Nutritivo, incubando a $37^{\circ} \mathrm{C} / 24$ horas. Se realizaron las pruebas de tinción de Gram, oxidasa y catalasa. Las cepas que fueron bacilos Gram positivos, catalasa positivas y oxidasa negativas fueron identificadas utilizando las siguientes pruebas:

- Movilidad: utilizando agar GI, incubando a $25^{\circ} \mathrm{C}$, durante siete días y haciendo lecturas diarias del crecimiento en sombrilla, típicas de la mayoría de las especies de Listeria.

- Fermentación de carbohidratos: se emplearon soluciones de D-manitol, ramnosa, y xilosa, esterilizadas por filtración y añadidas, hasta una concentración del $1,0 \%$, al caldo púrpura. Tras la inoculación de las cepas e incubación a $37^{\circ} \mathrm{C}$, se hizo la lectura a las 24 y a las $48 \mathrm{~h}$. Las cepas capaces de producir ácido a partir del carbohidrato respectivo viraron el color del indicador hacia amarillo. 
- Producción de $\beta$-hemólisis: se estudió la actividad $\beta$-hemolítica en placas de Agar Base Columbia, suplementado con sangre humana, al $5 \%$ no tratada, incubando a $37^{\circ} \mathrm{C}$, por $24-48 \mathrm{~h}$. Se consideraron positivas aquellas cepas que presentaron un halo de aclaramiento alrededor de la zona de siembra, como resultado de la lisis total de los eritrocitos, presentes en el medio.

- Prueba del CAMP: para esta prueba, se utilizaron cultivos de $24 \mathrm{~h}$, a $37^{\circ} \mathrm{C}$ de $\mathrm{S}$. aureus y Rhodococcus equi, empleando como medio de cultivo Agar Base Columbia al 5\%, de glóbulos rojos humanos. En cada placa de medio, se sembraron las cepas anteriores de forma vertical, con suficiente separación entre ellas para, posteriormente, sembrar las cepas a ensayar de forma horizontal, no permitiendo que se toquen unas con otras. Se incubaron a $37^{\circ} \mathrm{C}$, durante $24-48 \mathrm{~h}$. Para la lectura de la prueba, se examinaron las hemólisis en la zona de influencia de la siembra vertical.

Determinación de Escherichia coli: Se empleó la técnica del Número Más Probable (NMP), utilizando el caldo LMXFluorocult, de la siguiente manera: se realizaron diluciones seriadas de la muestra hasta $10^{-3}$, inocular $1 \mathrm{~mL}$ de cada una de las diluciones $10^{-1}, 10^{-2}$ y $10^{-3}$, en tres series de tres tubos de ensayo (tres tubos por cada dilución), conteniendo $10 \mathrm{~mL}$ del caldo e incubando a $37^{\circ} \mathrm{C}$, por $24 \mathrm{~h}$. Se realizó la lectura de $E$. coli teniendo en cuenta la aparición de un color azul en los tubos, además, de fluorescencia y de un anillo rojo, luego de adicionar el reactivo de Kovac.

Para el análisis estadístico, se empleó el software estadístico SPSS. Se aplicó la prueba chi-cuadrado de Pearson, para probar si existían diferencias significativas entre los métodos empleados para caracterizar las especies de Listeria, método tradicional e inmunocromatográfico. Finalmente, se empleó la correlación de Spearman con $\mathrm{p}<0,05$, para determinar el grado de asociación entre Listeria y la presencia de coliformes fecales en las muestras.

\section{RESULTADOS Y DISCUSIÓN}

Para la adscripción a especie de las cepas de Listeria, se siguieron los lineamientos estipulados por NTC 4666; en la figura 1 , se especifican las diferentes especies aisladas y su método de aislamiento. Se aislaron dos cepas de Listeria monocytogenes, lo que indica una prevalencia del 3,9\% (las dos detectadas por el método inmunocromatográfico); aunque esta prevalencia es baja, se debe considerar desde el punto de vista de la salud pública, ya que todas las cepas de L. monocytogenes son patógenas para el hombre (Norrung, 2000). La incidencia de $L$. monocytogenes detectada aquí es similar a la hallada Markkula et al. (2005), quienes encontraron una prevalencia del $4,0 \%$, a partir de muestras de trucha arcoíris fresca y congelada y Rodas-Suárez et al.
(2006) reportaron una prevalencia por esta bacteria, de 4,5\% en pescado procedente de Méjico; Martínez \& Villalobos (2004) analizando muestras de atún fresco procedente de Venezuela, hallaron esta bacteria, con una prevalencia del 3,75\%; Miya et al. (2010), en el 2,6\% de muestras de bloques de atún; en Italia, Busani et al. (2005) analizaron una gran variedad de alimentos de origen animal, entre ellos pescados y derivados, logrando detectar esta bacteria en el $6,4 \%$, de muestras de pescado y sus derivados, destacando que fue de las más altas tasas de aislamiento, en comparación con otros alimentos. Por otro lado, Al Bulushi et al. (2010), no lograron aislar esta bacteria en muestras de pescado de origen marino.

Los datos hallados aquí son inferiores a los reportados por otros autores: Herrera et al. (2006) encontraron una incidencia del $10 \%$, para $L$. monocytogenes, en pescado de origen marino; Pao et al. (2008) reportaron una prevalencia del $12,5 \%$, en pescado procedente de piscifactorías; Chou et al. (2006), del 37,4\%, a partir de muestras de filetes de bagre y Yucel \& Balci (2010), del $44,5 \%$, en pescado de agua dulce, procedente de mercados en Turquía. En general, las prevalencias reportadas de $L$. monocytogenes en países tropicales son mucho menores que las obtenidas en países templados (Karunasagar \& Karunasagar, 2000), lo que se puede atribuir, principalmente, a la temperatura del agua. Como se mencionó anteriormente, L. monocytogenes es una bacteria psicrotrófica, de tal manera que las bajas temperaturas de los hábitats de peces procedentes de países europeos favorecen la multiplicación de este microorganismo, contrario a lo que sucede en países tropicales, como el nuestro, en el que las temperaturas más elevadas de los hábitats de los peces tienden a inhibirla.

Adicionalmente, se aislaron nueve cepas de L. innocua (Figura 1), lo que corresponde a una prevalencia del $17,6 \%$, (55,5\% recuperadas por el método tradicional y el $44,5 \%$ por el inmunocromatográfico); esta prevalencia, se relaciona con la encontrada por otros autores: $19 \%$, reportada por Yucel \& Balci (2010), en muestras de pescado de agua dulce; $20,6 \%$, encontrada por Chou et al. (2006), en muestras de filetes de bagre; 15\%, obtenida por Pao et al. (2008), en filetes procedentes de acuicultivos. Por otro lado, Rodas-Suárez et al. (2006) encontraron una prevalencia de $L$. innocua mucho mayor del $44 \%$, en pescados de origen marino; Miettinen \& Wirtanen (2005) reportaron prevalencia por esta especie, del 33\%, a partir de lotes de piscifactorías de trucha arcoíris fresca. Estas diferencias se pueden explicar, por el tipo de muestra analizada; los otros autores mencionados analizaron muestras de peces que incluían vísceras, branquias, piel, en las que, obviamente, se incrementa la microbiota. En el estudio que nos ocupa, se analizó únicamente la parte comestible del pescado; en todo caso, es preferible encontrar prevalencias altas de esta bacteria que se considera apatógena para el hombre (Jay et al. 2009). 


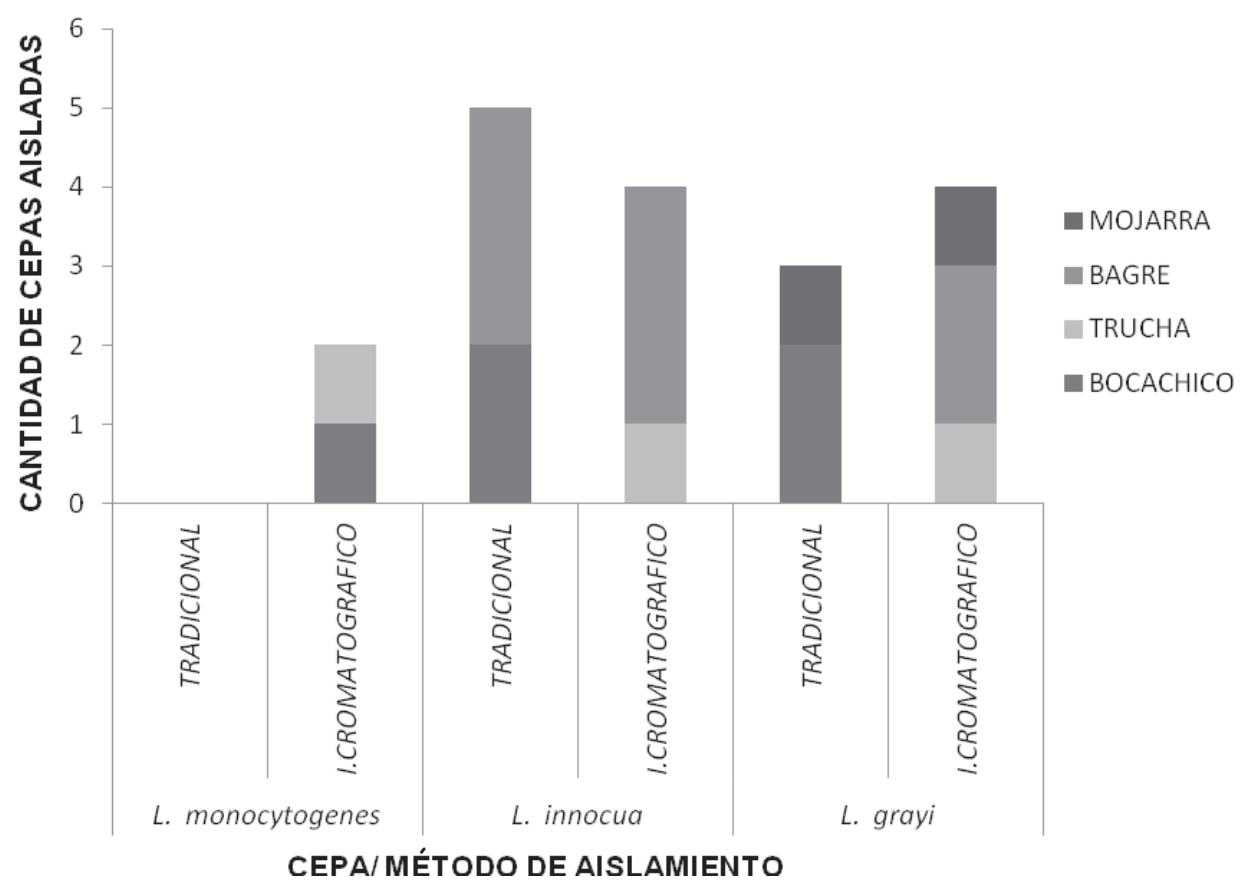

Figura 1: Cepas de Listeria spp. aisladas y método de detección.

Se aislaron siete cepas L. grayi (Figura 1), correspondiendo a una prevalencia del $13,7 \%$ (34,8\% recuperadas por el método tradicional y el $57,2 \%$ de por el inmunocromatográfico). Jallewar et al. (2007) encontraron una prevalencia por esta bacteria del 8,0\%, en pescado de agua dulce; Martínez \& Villalobos (2004), analizando muestras de atún fresco procedente de Venezuela, hallaron esta bacteria con una prevalencia del 2,5\%; Chou et al. (2006) reportaron una prevalencia del $0,8 \%$, a partir de muestras de filetes de bagre.

En total, la prevalencia de cepas de Listeria spp., en las muestras de pescado analizadas fue muy alta: $35,3 \%$, dato que se relaciona con el reportado por Miettinen \& Wirtanen (2005), del 35\%, en lotes de piscifactorías de trucha arcoíris fresca no procesada; Pao et al. (2008) reportaron prevalencias de Listeria, en filetes de pescado, del $27 \%$; Rodas-Suárez (2006), del 22,7\%, en muestras de pescado en Méjico; no obstante, Souza et al. (2008) y Morales et al. (2004) no detectaron Listeria spp. en muestras de pescado procedente de Brasil y de Costa Rica, respectivamente. Las diferencias en los resultados obtenidos, se pueden deber a la contaminación de las aguas donde se desarrollan estos animales; al respecto es muy importante considerar que la mayoría de los pescados analizados en este estudio procedían del río Magdalena que, como es bien sabido, es un colector de aguas residuales domésticas e industriales de diferente índole; también es necesario considerar la contaminación adicional que se aporta a este alimento, durante las operaciones de transporte, de distribución y de venta.
Al comparar estadísticamente, mediante la prueba Chicuadrado de Pearson, los dos métodos empleados para todas las muestras analizadas, tradicional e inmunocromatográfico, se encontró que no existían diferencias significativas entre los dos métodos $(\mathrm{p}>0,05)$.

La presencia de Listeria spp. puede ser indicadora de deficientes prácticas higiénico-sanitarias en el procesamiento de los alimentos (Windrantz \& Arias, 2000), de tal manera que la higiene personal y programas de limpieza y de desinfección adecuados, podrían ser suficientes para controlar Listeria spp. (Gibbons et al. 2006; Keeratipibul \& Lekroengsin, 2009). La implementación de sistemas de control de calidad en las industrias procesadoras de pescado, como el HACCP, es de vital importancia, para asegurar el control de este patógeno (Destro, 2000); finalmente, la adecuada y la suficiente cocción del pescado antes de su consumo reduce el riesgo por esta bacteria, ya que, como se mencionó anteriormente, L. monocytogenes es termosensible.

Esta establecido que la contaminación del pescado con $L$. monocytogenes se origina, principalmente, en los equipos, en los utensilios, en las superficies, en los manipuladores y no tanto en la materia prima, como tal (Chen et al. 2010b); Hansen et al. (2006) encontraron que entre mayor contacto con el hombre tenga el pescado mayor es la presencia de $L$. monocytogenes en los ambientes de procesamiento, llegando a ser del $10 \%$ en piscifactorias de agua dulce. Es evidente que inadecuadas prácticas de limpieza y de desinfección 
aumentan la persistencia de la bacteria en los equipos y en los ambientes de procesamiento (Carpentier \& Cerf, 2011; Chen et al. 2010c); a esto se debe sumar la demostrada resistencia de L. monocytogenes a desinfectantes, cuando forma biopelículas (Folsom \& Frank, 2006).

Es importante recalcar que, dada la capacidad de $L$. monocytogenes de crecer a temperaturas de refrigeración, la presencia de esta bacteria, aún a bajas concentraciones, representa un riesgo para la salud pública cuando el pescado se mantiene a estas temperaturas durante largos periodos de tiempo (Chou et al. 2006); para ilustrar lo anterior, en el $12 \%$ de muestras de pescados o derivados tomadas de refrigeradores de pacientes con listeriosis, se recuperó L.monocytogenes, consiguiéndose aislar el mismo serotipo en el paciente (Rocourt et al. 2000).

Otro aspecto importante a considerar es la probabilidad que la bacteria resista los tratamientos térmicos cuando la concentración inicial es muy alta $\left(10^{5}-10^{6} \mathrm{ufc} / \mathrm{g}\right)$ (ICMSF, 1998); aunque L. monocytogenes se considera termosensible, se ha encontrado en pescados tratados por calor, enfatizando la posible contaminación cruzada postproceso (Huss et al. 2003; Chou et al. 2006).

En cuanto a la presencia de $E$. coli en las muestras analizadas, se determinó que no existe correlación entre el NMP de $E$. coli con la presencia de Listeria spp. en las muestras, en el sentido que puede existir presencia de E.coli y ausencia de Listeria (Tabla 1). Al comparar los resultados obtenidos para E.coli con las recomendaciones establecidas por la ICMSF para pescado fresco, se tiene que el $8 \%$ de las muestras analizadas se consideran inaceptables (Tabla 2); Morales et al. (2004) encontraron que el 58\% de las muestras de tilapia procedentes de acuicultivos eran inaceptables, según los parámetros recomendados por la ICMSF para E. coli; por otro lado, Pao et al. (2008) determinaron que ninguna de las muestras de filetes de pescado procedentes de acuicultivos superaban los parámetros de calidad marginalmente aceptables para E. coli, estipulados por la ICMSF. Estas diferencias se podrían explicar bien sea por aguas de crianza

Tabla 1: Presencia de Escherichia coli y Listeria spp. en las muestras de pescado analizadas. contaminadas, como se mencionó anteriormente; la mayoría de los pescados analizados provenían del río Magdalena o por prácticas post-captura deficientes, concretamente, medidas higiénicas deficientes, durante la manipulación del pescado, utilización de agua de mala calidad en la elaboración del hielo empleado para mantener frío el pescado o abusos de temperatura durante su conservación (Pamuk et al. 2011; ICMSF, 1999).

En conclusión, se lograron aislar e identificar cepas de $L$. monocytogenes, L. innocua y L. grayi, a partir de muestras de pescado fresco, comercializado en la plaza de mercado en Pamplona; no se encontraron diferencias significativas entre los dos métodos de detección empleados, razón por la cual, se considera que es factible emplear indistintamente estos métodos para la detección de la bacteria; finalmente, la presencia de $E$. coli es un buen indicador de la presencia de cepas de Listeria, en las muestras analizadas.

La presencia de cepas de Listeria en las muestras refleja, probablemente, las inadecuadas prácticas de higiene y de desinfección empleadas a lo largo de toda la cadena de procesamiento del mismo, de tal manera que se debe capacitar a los manipuladores, a los consumidores y a los productores de este alimento, en la implementación de Buenas Prácticas de Manufactura. A este respecto, se recomienda a la oficina de Saneamiento Ambiental del municipio de Pamplona, realizar seguimientos y verificaciones de las medidas higiénicas que se aplican en los puntos de venta de pescado del mercado de la ciudad.

Es importante recalcar que las diferencias en los resultados encontrados aquí con los hallados por otros autores, se pueden deber a la metodología utilizada, a diferencias en el tipo de muestra analizada (pescado fresco y fileteado), el origen (agua dulce o de mar), a variaciones debidas a las distintas especies de peces, a la estación del año o a las condiciones higiénico-sanitarias empleadas, durante el procesamiento del pescado.

Finalmente, sería convenienterealizarpruebas deconfirmación de las cepas aisladas, mediante pruebas genotípicas, como

\begin{tabular}{|l|c|c|}
\hline PESCADO & E.coli $(\%)^{1}$ & Listeria spp. (\%) $^{2}$ \\
\hline Bagre & 95 & 15,7 \\
\hline Mojarra & 71,4 & 3,9 \\
\hline Bocachico & 100 & 9,8 \\
\hline Trucha & 50 & 5,9 \\
\hline
\end{tabular}

${ }^{1}$ : NMP mayor o igual a 3

${ }^{2}$ : independientemente del método de detección 
Tabla 2: Número Más Probable (NMP) de Escherichia coli en las muestras de pescado analizadas.

\begin{tabular}{|c|c|c|}
\hline NMP E.coli/g & \% MUESTRAS & CRITERIO DE CALIDAD $^{1}$ \\
\hline$<3-11$ & 37 & Buena \\
\hline$>11-500$ & 55 & Marginalmente aceptable \\
\hline$>500$ & 8 & Inaceptable \\
\hline
\end{tabular}

${ }^{1}$ : según ICMSF (1999)

la Reacción en Cadena de la Polimerasa (PCR), buscando, para el caso específico de $L$. monocytogenes, genes que codifiquen factores de virulencia, como son el gen de la internalina (gen inlA, hemolisina y/o la listeriolisina (gen hly) y la proteína p60 (gen iap).

Agradecimientos: A la Universidad de Pamplona, Facultad de Ciencias Básicas, Departamento de Microbiología, Grupo de Investigación en Microbiología y Biotecnología (GIMBIO) y su semillero. Conflictos de intereses: El manuscrito fue preparado y revisado con la participación de todos los autores, quienes declaramos que no existe ningún conflicto de intereses que ponga en riesgo la validez de los resultados presentados. Financiación: Este estudio fue financiado por el Grupo de Investigación en Microbiología y Biotecnología (GIMBIO) Universidad de Pamplona.

\section{BIBLIOGRAFÍA}

1. AL BULUSHI, I.; POOLE, S.; BARLOW, R.; DEETH, H.; DYKES, G. 2010. Speciation of Gram-positive bacteria in fresh and ambient-stored sub-tropical marine fish. Int. J. Food Microbiol. 138:32-38.

2. BUSANI, L.; CIGLIANO, A.; TAIOLI, E.; CALIGIURI, V.; CHIAVACCI, L.; DI BELLA, C.; BATTISTI, A.; DURANTI, A.; GIANFRANCESCHI, M.; NARDELLA, M.C.; RICCI, A.; ROLESU, S.; TAMBA, M.; MARABELLI, R.; CAPRIOLI A. 2005. Prevalence of Salmonella enterica and Listeria monocytogenes contamination in foods of animal origin in Italy. J. Food Prot. 68(8):1729-1733.

3. CARPENTIER, B.; CERF, O. 2011.Review-Persistence of Listeria monocytogenes in food industry equipment and Premises. Int. J. Food Microbiol. 145:1-8.

4. CRESPO, M.; VÉLEZ, J.; CASTAÑEDA, C.; HOYOS, F.; LÓPEZ, M.; SALAZAR, J. 1999. Aislamiento de
Listeria monocytogenes en un hospital de tercer nivel. Colombia Médica. 30:89-90.

5. CHEN, B.; PYLA, R.; KIM, T.; SILVA, J.; JUNG, Y. 2010a. Antibiotic resistance in Listeria species isolated from catfish fillets and processing environment. Lett. Appl. Microbiol. 50:626-632.

6. CHEN, B.; PYLA, R.; KIM, T.; SILVA, J.; JUNG, Y. 2010b. Prevalence and contamination patterns of Listeria monocytogenes in catfish processing environment and fresh fillets. Food Microbiol. 27:645-652.

7. CHEN, B.; PYLA, R.; KIM, T.; SILVA, J.; JUNG, Y. 2010c. Incidence and persistence of Listeria monocytogenes in the Catfish processing environment and fresh fillets. J. Food Prot. 73(9):1641-1650.

8. CHOU, CH.; SILVA, J.; WANG, C. 2006. Prevalence and typing of Listeria monocytogenes in raw catfish fillets. J. Food Prot. 69(4):815-819.

9. DESTRO, M. 2000. Incidence and significance of Listeria in fish and fish products from Latin America. Int. J. Food Microbiol. 62:191-196.

10. ELLIOT, E.; KVENBERG, J. 2000. Risk assessment used to evaluate the US position on Listeria monocytogenes in seafood. Int. J. Food Microbiol. 62:253-260.

11. FOLSOM, J.; FRANK, J. 2006. Chlorine resistance of Listeria monocytogenes biofilms and relationship to subtype, cell density, and planktonic cell chlorine resistance. J. Food Prot. 69(6):1292-6.

12. GIBBONS, I.; ADESIYUNA, A.; SEEPERSADSINGHA, N.; RAHAMAN, S. 2006. Investigation for possible source(s) of contamination of ready-to-eat meat 
products with Listeria spp. and other pathogens in a meat processing plant in Trinidad. Food Microbiol. 23:359-366.

13. HANSEN, C.; VOGEL, B.; GRAM, L. 2006. Prevalence and survival of Listeria monocytogenes in Danish aquatic and fish-processing environments. J. Food Prot. 69(9):2113-2122.

14. HERRERA, F.; SANTOS, J.; OTERO, A.; GARCÍALÓPEZ, M. 2006. Ocurrence of foodbone pathogenic bacteria in displayed portions of marine fish. J. Appl. Microbiol. 100: 527-536.

15. HUSS, H.; ABABOUCH, L.; GRAM, L. 2003. Assessment and Management of Seafood Safety and Quality. Rome: Disponible desde Internet en: FAO.ftp://ftp. fao.org/docrep/fao/006/y4743e/y4743e00.pdf (con acceso 29/01/12).

16. ICMSF. 1998. Microorganismos de los alimentos 5: Características de los patógenos microbianos. Editorial Acribia. Zaragoza. 170p.

17. ICMSF. 1999. Microorganismos de los alimentos 2: Métodos de muestreo para análisis microbiológicos: principios y aplicaciones específicas. Editorial Acribia. Zaragoza. 171p.

18. JALLEWAR, P.; KALOREY, D.; KURKURE, N.; PANDE, V.; BARBUDDHE, S. 2007. Genotypic characterization of Listeria spp. isolated from fresh water fish. Int. J. Food Microbiol. 114:120-123.

19. JAY, J.; LOESSNER, M.; GOLDEN, D. 2009. Microbiología Moderna de los Alimentos. Ed. Acribia. Zaragoza. 477, 599p.

20. KARUNASAGAR, I.; KARUNASAGAR, I. 2000. Listeria in tropical fish and fishery products. Int. J. Food Microbiol. 62:177-181.

21. KEERATIPIBUL, S.; LEKROENGSIN, S. 2009. Risk analysis of Listeria spp. contamination in two types of ready-to-eat chicken meat products. J. Food Prot. 72(1):67-74.

22. LOMONACO, S.; NUCERA, D.; PARISI, A.; NORMANNO, G.; BOTTERO, M. 2011. Comparison of two AFLP methods and PFGE using strains of Listeria monocytogenes isolated from environmental and food samples obtained from Piedmont, Italy. Int. J. Food Microbiol. 149:177-182.
23. MARÍN, C.; FONSECA, C.; ARIAS, S.; VILLEGAS, I.; GARCÍA, A.; ISHIHARA, H. 2009. Carga bacteriana de los peces Cynoscion squamipinnis (Perciformes: Scianidae) y Lutjanus gutattus (Perciformes: Lutjanidae) en la cadena de comercialización, Costa Rica. Rev. Biol. Trop. 57 (1-2):45-52.

24. MARKKULA, A.; AUTIO, T.; LUNDÉN, J.; KORKEALA, H. 2005. Raw and processed fish show identical Listeria monocytogenes genotypes with pulsed-field gel electrophoresis. J. Food Prot. 68(6):1228-1231.

25. MARTíNEZ, R.; VILLALOBOS, L. 2004. Aislamiento de Listeria monocytogenes en atún fresco expedido en la ciudad de Cumaná, Venezuela. Revista Científica, FCV-LUZ. 14(4):354-357.

26. MEDRANO, M.; RESTREPO, S.; VANEGAS, C. 2006. Tipificación molecular de Listeria monocytogenes aisladas de muestras clínicas y alimentos. Biomédica. 26 (3):442-450.

27. McMEEKIN, T. 2003. Detecting pathogens in foods. Cambridge-England:Woodhead Publishing Limited. 220p. Disponible desde Internet en: http://books. google.com/books?id=SKQ7DJd7-h0C\&pg=PA22 2\&lpg $=$ PA223\&ots $=$ ds75PQhcub\&dq $=$ detection + of + listeria $+\mathrm{in}+$ foods + by + singlepath $\& \mathrm{hl}=\mathrm{es} \# \mathrm{v}=$ onepage \& q = detection\%20of\%20listeria\%20in\%20 foods $\% 20$ by $\% 20$ singlepath $\& \mathrm{f}=$ false $\quad$ (con acceso 03/02/2012).

28. MERCK. 2008. GLISA-Rapid Test (Gold Labelled ImmunoSorbent Assay) for the qualitative detection of Listeria spp. in food and environmental samples. Disponible desde Internet en: http://www.merckchemicals.com/spain/singlepath-listeria/MDA CHEM-104142/p_qFGb.s1LXiIAAAEWPuEfVhTI?att achments $=$ TI (con acceso 15/03/2012).

29. MieTtinen, H.; WIRTANEN, G. 2005. Prevalence and location of Listeria monocytogenes in farmed rainbow trout. Int. J. Food Microbiol. 104:135-143.

30. MIYA, S.; TAKAHASHI, H.; ISHIKAWA, T.; FUJII, T.; KIMURA, B. 2010. Risk of Listeria monocytogenes contamination of raw ready-to-eat seafood products available at retail outlets in Japan. Appl. Environ. Microbiol. 76(10):3383-3386.

31. MORALES, G.; BLANCO, L.; ARIAS, M.; CHAVES, C. 2004. Evaluación de la calidad bacteriológica de tilapia fresca (Orochoromis niloticus) proveniente de 
la zona norte de Costa Rica. Arch. Latinoam. Nutr. 54(4):433-437.

32. MOTARJEMI, Y.; ADAMS, M. 2006. Emerging Foodborne Pathogens. Woodhead Publishing Limited. Cambridge England. 416,420p.

33. MURIEL, M. 2008. Estimación de la Incidencia de las enfermedades transmitidas por alimentos (ETA) en Colombia en la década 1996-2006. Trabajo de grado. Pontificia Universidad Javeriana. 2008. Disponible desde Internet en: http://www.javeriana. edu.co/biblos/tesis/ciencias/tesis134.pdf (con acceso 15/03/2012).

34. NORMA TÉCNICA COLOMBIANA 4666. 1999. Método Horizontal para la detección de Listeria monocytogenes. ICONTEC.

35. NORRUNG, B. 2000. Microbiological criteria for Listeria monocytogenes in foods under special consideration of risk assessment approaches. Int. J. Food Microbiol. 62:217-221.

36. ORSI, R.; DEN BAKKER, H.; WIEDMANN, M. 2011. Listeria monocytogenes lineages: Genomics, evolution, ecology, and phenotypic Characteristics. Int. J. Med. Microbiol. 301:79-96.

37. OTTO, R.; PAKER, I.; BANE, L.; BEAMER, S.; JACZYNSKI, J.; MATAK, K. 2011. Survival of Listeria innocua in rainbow trout protein recovered by isoelectric solubilization and precipitation with acetic and citric acids. J. Food Prot. 74(8):1348-1352.

38. PAMUK, S.; GURLER, Z.; YILDIRIM, Y.; SIRIKEN, B. 2011. Detection of microbiological quality of common carp (Cyprinus carpio) sold in public bazaar in Afyonkarahisar. J. Anim. Vet. Adv. 10(8):1012-1018.

39. PAO, S.; ETTINGER, M.; KHALID, M.; REID, A.; NERRIE, B. 2008. Microbial quality of raw aquacultured fish fillets procured from Internet and local retail markets. J. Food Prot. 71(8):1544-9.
40. ROCOURT, J.; JACQUET, CH.; REILLY, A. 2000. Epidemiology of human listeriosis and seafoods. Int. J. Food Microbiol. 62:197-209.

41. RODAS-SUÁREZ, O.; FLORES-PEDROCHE, J.; BETANCOURT-RULE, J.; QUIÑONES-RAMÍREZ, E.; VÁZQUEZ-SALINAS, C. 2006. Occurrence and antibiotic sensitivity of Listeria monocytogenes strains isolated from oysters, fish, and estuarine water. Appl. Environ. Microbiol. 72(11):7410-7412.

42. ROSS, T.; DALGAARD, P.; TIENUINGOON, S. 2000. Predictive modelling of the growth and survival of Listeria in fishery products. Int. J. Food Microbiol. 62:231-245.

43. SOUZA, V.; ALVES, V.; DESTRO, M.; DE MARTINIS, E. 2008. Quantitative evaluation of Listeria monocytogenes in fresh and processed surubim fish (Pseudoplatystoma sp). Braz. J. Microbiol. 39:527528.

44. TODD, E.; NOTERMANS, S. 2011. Surveillance of listeriosis and its causative pathogen, Listeria monocytogenes. Food Control 22:1484-1490.

45. WAN NORHANA, M.; POOLE, S.; DEETH, H.; DYKES, G. 2010. Prevalence, persistence and control of Salmonella and Listeria in shrimp and shrimp products: A review. Food Control 21:343-361.

46. WINDRANTZ, P.; ARIAS, M. 2000. Evaluation of the bacteriological quality of ice cream sold at San Jose, Costa Rica. ALAN. 50(3):301-303.

47. YUCEL, N.; BALCI, S. 2010. Prevalence of Listeria, Aeromonas, and Vibrio species in fish used for human consumption in Turkey. J. Food Prot. 73(2):380-384.

Recibido: Febrero 6 de 2012

Aceptado: Agosto 13 de 2012 